\title{
Gait Parameters and Muscle Activation Patterns at 3, 6 and 12 Months After Total Hip Arthroplasty
}

\author{
Valentina Agostini, PhD ${ }^{\mathrm{a}}$, Daria Ganio, MD ${ }^{\mathrm{b}}$, Katia Facchin, PT ${ }^{\mathrm{b}}$, Luciano Cane, MD ${ }^{\mathrm{b}}$, \\ Susana Moreira Carneiro, MSc ${ }^{c}$, Marco Knaflitz, $\mathrm{PhD}^{\mathrm{a}}$ \\ a Department of Electronics and Telecommunications, Politecnico di Torino, Torino, Italy \\ b Gait Laboratory Rehabilitation Unit, ASLTO4 Piemonte, Ivrea, Italy \\ c Politécnico de Bragança, Bragança, Portugal
}

\section{A R T I C L E I N F O}

\section{Article history:}

Received 16 July 2013

Accepted 11 December 2013

\section{Keywords:}

total hip arthroplasty

hip replacement

gait

electromyography

follow-up

\begin{abstract}
A B S T R A C T
Gait analysis was performed on 20 patients with unilateral hip prosthesis (3, 6 and 12 months postoperatively) and 20 controls to investigate their gait characteristics and muscle activation patterns. One year after the intervention, patients still walked with a higher percentage of "atypical" cycles, a prolonged heel contact, a shortened flat foot contact, a reduced hip dynamic range of motion and abnormal timing in the muscle activation patterns of tibialis anterior, gastrocnemius lateralis, biceps femoris and gluteus medius, with respect to the control group. Although the gait velocity and the knee range of motion improved from 3 to 6 months post-surgery, the above mentioned parameters did not improve from 6 to 12 months. THA patients failed to obtain normal gait one year after surgery.
\end{abstract}

(c) 2014 Elsevier Inc. All rights reserved.
Total hip arthroplasty (THA) is a surgical procedure indicated for patients affected by severe osteoarthritis of the hip [1]. Despite a surgeon's ability, THA inevitably damages muscles and peri-articular tendons and brings forth a loss of joint proprioceptors [2]. Literature reports that the surgical insult, deconditioning and compensatory mechanisms adopted by the patient in the rehabilitation period may result in residual impairment, with a decline of hip abductor function $[3,4]$ and gait symmetry [5].

Gait analysis is increasingly used to quantitatively assess a patient's functional improvement and to evaluate hip surgery procedures [6-8] and implant types [9-12]. Some studies considered surface electromyography (EMG) as an adjunctive tool that complements gait analysis by monitoring muscle activity during gait [12-15]. However, these studies limited the EMG analysis to a few gait cycles for each assessment session. This prevented researchers from analyzing the different muscle activation patterns of human gait. These patterns can be reliably observed by recording a subject's gait for at least 2-3 min, thus collecting a sufficiently large number (100200) of consecutive strides for applying a statistical approach to data analysis. 'Statistical gait analysis' was developed to obtain gait parameters and muscle activation patterns by analyzing a large number of strides in a user independent manner [16-18].

The Conflict of Interest statement associated with this article can be found at http:// dx.doi.org/10.1016/j.arth.2013.12.018.

Reprint requests: Valentina Agostini, PhD, Department of Electronics and Telecommunications, Politecnico di Torino, Corso Duca degli Abruzzi 24, 10129 Torino, Italy.
The present study tests the null hypothesis that there are no gait differences between the control group and THA patients one year after surgery.

\section{Materials and Methods}

An a priori power calculation was performed to estimate the minimum sample size required for the study using data from the literature [13][19]. The hip dynamic range of motion (ROM) was chosen as the primary outcome measure for comparing the THA and control groups through a 1-tailed Student $t$-test, at a significance level $\alpha=0.05$ and power $=0.9$. The test was chosen 1 -tailed because we expected THA patients to show a hip dynamic ROM not greater than that of controls. We obtained a minimum sample size ranging from 7 to 10 patients, with an equal number of controls (allocation ratio 1:1).

\section{Participants}

This study analyzed 20 patients and 20 healthy controls. Patients were recruited from the Rehabilitation and Functional Recovery Unit at the Ivrea Hospital, Torino (Italy), between 2007 and 2009. They were enrolled in the study after the end of their (standard) rehabilitation protocol. Patients with bilateral coxoarthrosis, neurological problems and/or other orthopedic problems compromising gait were excluded from this study. We screened a total of 25 unilateral THA patients (see Fig. 1): 3 were excluded because they did not match the inclusions criteria (1 presented a reimplant, 1 was unable to cooperate due to cognitive deficits, 1 had a fracture in the 


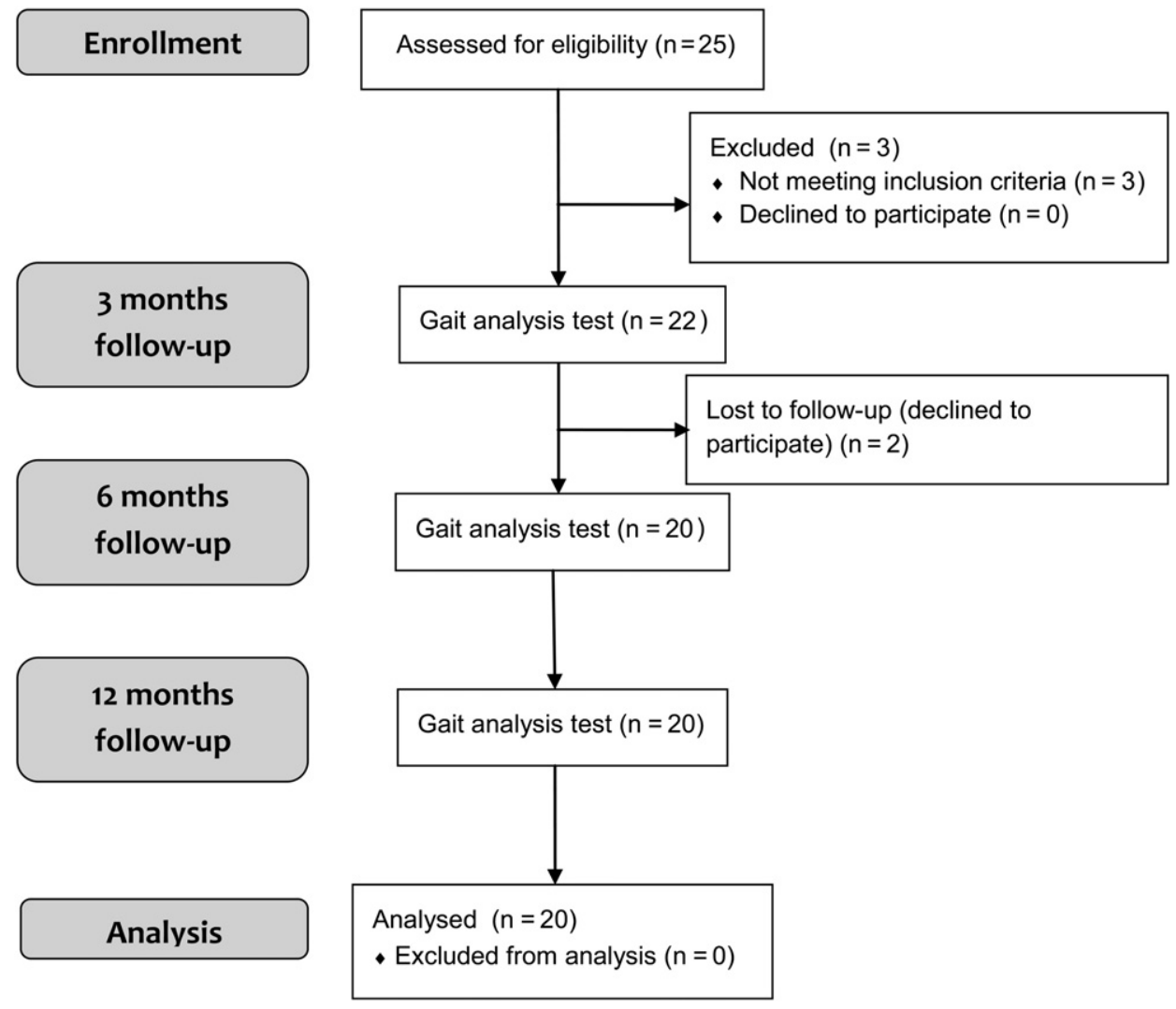

Fig. 1. Flow chart of the study.

contralateral acetabulum). Two patients declined to participate after the first gait test. Patients originally suffered from hip osteoarhtrosis and had surgery for primary unilateral THA, with posterior-lateral incision. After surgery, patients underwent joint and muscular rehabilitation. All patients received the same rehabilitation protocol. The load of the operated limb was gradually restored instructing the patients to first use two crutches, then partially loading the limb removing one crutch, and finally gaining full load. The whole rehabilitation program lasted 2 months. More specifically, patients were hospitalized 8-10 days for surgery, then they spent from 2 to 3 weeks in the Rehabilitation Unit, and, finally, they followed a onemonth rehabilitation program at their home. Controls were volunteers recruited from the local community in 2010. The authors feel that the THA and control populations are comparable due to the small difference in the period of time between the cohorts.

Both patients and controls underwent a physical examination prior to the gait analysis test and anthropometric data were collected. For patients, we also determined the leg length discrepancy three months after surgery, before running the first gait test, and we performed the longitudinal evaluation of the Harris hip score [20]. The leg length discrepancy was assessed with a tape measure from the anterior-superior iliac spine to the medial malleolus of each lower $\operatorname{limb}[21]$.

\section{Experimental Protocol}

The patients' outcome was assessed at 3, 6, and 12 months after surgery. Gait analysis was performed using a multichannel recording system (STEP32, DemItalia, Italy). Subjects were equipped bilaterally with: a) foot-switches under the heel, the first and fifth metatarsal heads, b) knee and hip joint goniometers (sagittal plane), and c) surface EMG electrodes positioned over the tibialis anterior, gastrocnemius lateralis, rectus femoris, biceps femoris and gluteus medius. We chose this EMG configuration to study at least a couple of flexor/ extensor muscles for each joint of the lower limb (ankle, knee, hip). Fig. 2 shows an instrumented subject. EMG electrodes were positioned according to the guidelines suggested by Winter [22]. Details on the EMG electrodes and the crosstalk issue are described in a previous work [16].

Subjects were instructed to walk barefoot at self-selected speed. They walked back and forth over a $10-\mathrm{m}$ pathway and each acquisition lasted $150 \mathrm{~s}$. The turns and the decelerations/acceleration phases in proximity of the turns were automatically removed and an actual straight path of $7 \mathrm{~m}$ was considered.

The experimental protocol was approved by the local ethical committee and all participants gave their written informed consent to be included in the study.

\section{Signal Processing}

The software of the acquisition system included the routines for statistical gait analysis. The following gait phases were obtained for each lower limb: heel contact (H), flat foot contact (F), push off (P), swing (S). Then, the signal was segmented in separate gait cycles and the different types of gait cycles found during a subject's walk were classified [17]. The cycles showing a 'normal' sequence of gait phases (HFPS) were extracted to be further processed. On the contrary, the cycles that did not match the normal sequence, called atypical cycles, were not considered in the evaluation of the gait parameters and EMG patterns. However, the percentage of atypical cycles occurred during a subject's walk was calculated, since previous studies revealed the importance of this parameter in evaluating gait performances [17].

The goniometric signal was low-pass filtered (FIR filter, 100 taps, cut-off frequency of $15 \mathrm{~Hz}$ ). The goniometric signal and the duration of the gait phases were used by a multivariate statistical filter (Hotelling t-test, $\alpha=0.05$ ) to discard outlier cycles, i.e., strides with the proper sequence of gait phases (HFPS) but with abnormal timing, like those relative to deceleration, reversing, and acceleration. 


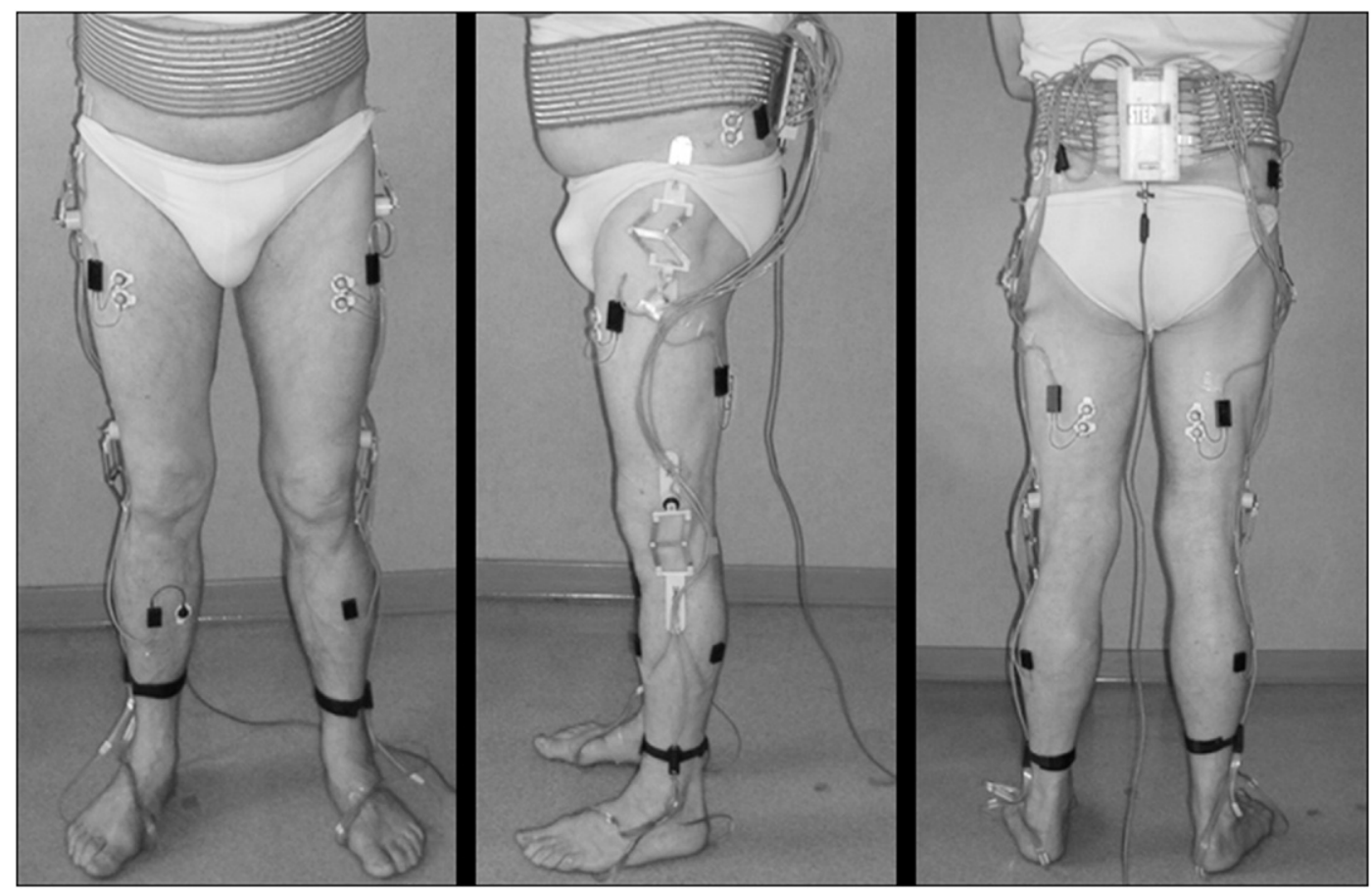

Fig. 2. Instrumented subject: front, side, and back view.

The EMG signal was high-pass filtered (FIR filter, 100 taps, cut-off frequency of $20 \mathrm{~Hz}$ ), and then processed by a double-threshold statistical detector to obtain, in a user-independent way, the muscle activation intervals [23].

\section{Data Analysis}

For each lower limb of a single subject, we collected a total of $145 \pm 25$ gait cycles (mean \pm SD). The following gait parameters were extracted: percentage of atypical cycles, velocity, period of double support (percentage of the gait cycle in which both feet are in contact with the ground), duration of each gait phase (percentage of the gait cycle (GC) spent in each of the three sub-phases of stance $\mathrm{H}, \mathrm{F}$, $\mathrm{P}$, and in swing $\mathrm{S}$ ), hip and knee dynamic range of motion. The dynamic range of motion (ROM) is defined as the difference between the maximum and the minimum value of the respective joint goniometric curve during gait.

Each muscle of a single subject showed different activation patterns while walking, as already documented in other studies $[16,24]$. We calculated the frequency of occurrence of strides showing $1,2,3,4$ or 5 muscle activations [16]. We limited the EMG analysis to the two most frequent activation patterns (those with the highest frequency of occurrence).

\section{Statistical Analysis}

All data distributions were tested for normality with a Kolmogorov-Smirnov test. For each of them, the null hypothesis could not be rejected at a significance level $\alpha=0.05$. Patients and controls were compared to determine if there were any significant differences in age, height and BMI with a Student t-test (two-sample, 2 tails, $\alpha=0.05)$. The Harris hip score of THA patients was compared between 3 and 6 months, and between 6 and 12 months post-surgery with a Student $t$-test (two-sample, 2 tails, $\alpha=0.05$ ). In order to establish if THA patients gained a normal walking pattern 1 year after surgery, we analyzed the group difference between the patients 12 months after surgery and controls. We applied a 1-factor multivariate analysis of variance (MANOVA) [25] on a set of 9 dependent variables (percentage of atypical cycles, velocity, double support, duration of the four gait phases, hip and knee dynamic ROM). More specifically, the MANOVA test was applied between the prosthetic side of patients and the right side of controls. This was chosen because for 14 patients out of 20 the prosthetic side was the right one. Furthermore, in order to evidence possible compensative strategies of the contralateral lower limb, we performed a second MANOVA test, on the same set of dependent variables, between the sound side of patients (at 12 months) and the left side of controls.

Student's $t$-tests (two-sample, $\alpha=0.05$ ) were used to explore the differences in the gait parameters between the groups. A1-tailed test was carried out for the velocity and dynamic ROMs, and a 2-tailed test for the other variables (atypical cycles, double support, gait phases). This was chosen because it is known from the literature that velocity in patients is not greater than that of controls, as well as their hip and knee dynamic ROMs, while there isn't any a priori knowledge on the other variables. Again the tests were performed between: 1) the prosthetic side of patients and the right side of controls, 2) the sound side of patients and the left side of controls.

After having studied the differences between patients ( 1 year after surgery) and controls, we concentrated on the THA within-group differences observed during the follow-up. Student's $t$-tests were used to explore the differences in the gait parameters between 3 and 6 months and between 6 and 12 months.

A post-hoc power analysis was performed on the statistically significant differences. The post-hoc analysis showed a power greater than 0.80 for all the parameters considered except for the percentage of atypical cycles (power equal to 0.38 ) and for the knee dynamic ROM (power equal to 0.79 ).

The statistical analysis was performed by means of custom routines written in MATLAB (The MathWorks Inc, Natick, MA).

\section{Results}

Participant characteristics are summarized in Table 1. No differences were found between the groups for age and height, however the THA group had a significant larger BMI ( $27.0 \pm 3.8$ vs. $23.9 \pm 2.8 \mathrm{~kg} /$ $\left.\mathrm{m}^{2} ; P=0.01\right)$. The THA group significantly increased the Harris hip 
Table 1

Anthropometric Characteristics of the Sample Populations (THA and Controls); Harris Hip Score of THA Patients.

\begin{tabular}{llllll}
\hline $\begin{array}{l}\text { Anthropometric } \\
\text { Characteristics }\end{array}$ & \multicolumn{2}{c}{$\begin{array}{c}\text { Hip Prosthesis Patients } \\
(\mathrm{N}=20)\end{array}$} & \multicolumn{2}{c}{ Controls $(\mathrm{N}=20)$} \\
\hline Age (years) & Mean (SD) & \multicolumn{2}{c}{$66.1(7.2)$} & \multicolumn{2}{c}{$65.4(5.1)$} \\
& Range & \multicolumn{2}{c}{$49-79$} & \multicolumn{2}{c}{$57-74$} \\
Gender & & 9 males & 11 females & 11 males & 9 females \\
Height $(\mathrm{cm})$ & Mean (SD) & $175.1(7.7)$ & $163.4(9.6)$ & $175.8(7.7)$ & $162.4(5.1)$ \\
& Range & $165-185$ & $150-179$ & $166-193$ & $155-170$ \\
Weight $(\mathrm{kg})$ & Mean (SD) & $80.2(10.7)$ & $74.3(15.0)$ & $76.1(11.1)$ & $60.3(6.6)$ \\
& Range & $60-92$ & $56-100$ & $60-96$ & $51-69$ \\
BMI $\left(\mathrm{kg} / \mathrm{m}^{2}\right)$ & Mean (SD) & $26.1(2.1)$ & $27.7(5.0)$ & $24.4(3.0)$ & $23.2(2.5)$ \\
& Range & $22.0-28.7$ & $20.9-34.5$ & $20.5-29.0$ & $19.1-26.3$
\end{tabular}

\begin{tabular}{lllll} 
& & \multicolumn{3}{c}{ Hip Prosthesis Patients $(\mathrm{N}=20)$} \\
\cline { 2 - 5 } THA Patients Characteristics & 3 Months & 6 Months & 12 Months \\
\hline \multirow{2}{*}{ Harris hip score } & Mean (SD) & $90.0(7.9)$ & $96.6(5.0)$ & $98.4(2.8)$ \\
& Range & $73-100$ & $83-100$ & $89-100$ \\
\hline
\end{tabular}

Abbreviation: BMI, Body Mass Index.

score between 3 and 6 months (90.0 \pm 7.9 vs. $96.6 \pm 5.0 ; P=$ $0.000004)$ and between 6 and 12 months ( $96.6 \pm 5.0$ vs. $98.4 \pm 2.8$; $P=0.02$ ). Note that although a statistical difference has been demonstrated for the Harris Hip score, this difference is below the threshold that can be detected by patients. The leg length discrepancy was $6 \pm 5 \mathrm{~mm}$ (range: $0-15 \mathrm{~mm}$ ).

\section{Between-Group Differences (12-Month THA vs. Control Group)}

One year after surgery, the THA group (prosthetic side) did not reach normal gait parameters compared to the control group (MANOVA Wilk's $\Lambda$ test: $P=0.007$ ). No significant differences were found for the sound side compared to the control group (MANOVA Wilk's $\Lambda$ test: $P=0.10$ ).

Gait parameters are summarized in Table 2. The 12-month THA group showed an increased percentage of atypical cycles $(15 \% \pm 11 \%$ vs. $9 \% \pm 6 \% ; P=0.04)$, an increased heel contact phase $(8.4 \% \pm 4.1 \%$ vs. $4.9 \% \pm 1.2 \%$ GC; $P=0.001)$, a reduced flat foot phase $(28.8 \% \pm$
$7.7 \%$ vs. $34.4 \% \pm 3.9 \% \mathrm{GC} ; P=0.01)$ and a reduced hip dynamic ROM $\left(16.3^{\circ} \pm 5.2^{\circ}\right.$ vs. $\left.19.2^{\circ} \pm 4.4^{\circ}, P=0.03\right)$. No significant differences were found for velocity, double support, push-off, swing and knee dynamic ROM compared to the control group.

Within-group differences (THA group at 3 vs. 6 months and at 6 vs. 12 months)

The THA group walked significantly slower at 3 months compared to 6 months post-surgery $(0.78 \pm 0.10$ vs. $0.92 \pm 0.18 \mathrm{~m} / \mathrm{s} ; P=$ $0.03)$ and with a lower knee dynamic ROM $\left(37.9^{\circ} \pm 7.3^{\circ}\right.$ vs. $41.8^{\circ} \pm$ $\left.6.8^{\circ} ; P=0.04\right)$. No significant differences were found for atypical cycles, velocity, gait phases and hip dynamic ROM. The THA group did not show significant improvements between 6 and 12 months for any of the examined gait parameters, although the percentage of atypical cycles and hip dynamic ROM showed $P$-values at the limits of significance $(P=0.054$ and $P=0.051$, respectively).

\section{Joint Kinematics}

Knee and hip joint angular motion is presented in Fig. 3 for the THA group at 3, 6 and 12 months post-surgery. The standard deviation limits of the control group are shown superimposed. The prosthetic side of the THA group showed a limited knee extension in stance, between the 1 st and the 2nd arc of flexion, throughout the follow-up. Three months after surgery it is also evident the limited knee dynamic ROM compared to the control group. The prosthetic side of the THA group also showed a limited hip extension compared to the control group. The hip joint curve of the THA group gradually improved during the follow-up, although it did not reach normality at 12 months post-surgery. The sound side of the THA group did not show noticeable differences in the knee and hip joint kinematics compared to the control group, except for a slight elevation of the entire knee curve towards higher values of the knee flexion.

\section{Muscle Activation Timing}

The muscle activation intervals are presented in Fig. 4. In each panel we show the two most frequent activation patterns. In tibialis

Table 2

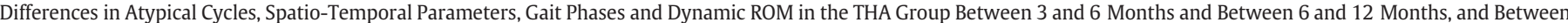
Controls and the THA Group 12 Months After Surgery.

\begin{tabular}{|c|c|c|c|c|c|c|c|c|c|}
\hline \multicolumn{8}{|c|}{ Hip Prosthesis Patients } & \multirow{2}{*}{\multicolumn{2}{|c|}{ Controls }} \\
\hline & & 3 Months & $P$ Value $^{\mathrm{a}}$ & 6 Months & $P$ Value $^{\mathrm{b}}$ & 12 Months & $P$ Value $^{\mathrm{c}}$ & & \\
\hline \multirow[t]{2}{*}{ Atypical cycles (\%) } & PR & $16(12)$ & 0.12 & $24(17)$ & 0.05 & $15(11)$ & 0.04 & $9(6)$ & $\mathrm{R}$ \\
\hline & SO & $17(11)$ & 0.76 & $18(7)$ & 0.17 & $15(8)$ & 0.06 & $10(9)$ & $\mathrm{L}$ \\
\hline \multicolumn{10}{|c|}{ Spatio-temporal parameters } \\
\hline Velocity $(\mathrm{m} / \mathrm{s})$ & & $0.78(0.10)$ & 0.03 & $0.92(0.18)$ & 0.19 & $1.00(0.22)$ & 0.45 & $0.99(0.17)$ & \\
\hline Double support (\% GC) & & $19.9(5.8)$ & 0.34 & $17.9(7.3)$ & 0.52 & $16.3(7.8)$ & 0.66 & $17.2(3.4)$ & \\
\hline \multicolumn{10}{|l|}{ Gait phases } \\
\hline \multirow[t]{2}{*}{ H, Heel contact (\% GC) } & PR & $9.3(6.7)$ & 0.68 & $8.5(4.5)$ & 0.90 & $8.4(4.1)$ & 0.001 & $4.9(1.2)$ & $\mathrm{R}$ \\
\hline & SO & $8.1(3.9)$ & 0.48 & $8.9(3.7)$ & 0.21 & $7.4(3.6)$ & 0.01 & $5.1(1.1)$ & $\mathrm{L}$ \\
\hline \multirow[t]{2}{*}{ F, Flat foot (\% GC) } & PR & $31.7(8.5)$ & 0.29 & $28.8(8.2)$ & 0.98 & $28.8(7.7)$ & 0.01 & 34.4 (3.9) & $\mathrm{R}$ \\
\hline & SO & $30.6(7.0)$ & 0.28 & $27.9(8.6)$ & 0.69 & $29.0(8.4)$ & 0.01 & $34.1(2.8)$ & $\mathrm{L}$ \\
\hline \multirow[t]{2}{*}{ P, Push off (\% GC) } & PR & $18.7(4.0)$ & 0.18 & $20.9(6.0)$ & 0.96 & $20.9(3.4)$ & 0.06 & $18.9(3.3)$ & $\mathrm{R}$ \\
\hline & SO & $21.7(6.5)$ & 0.59 & $22.8(6.0)$ & 0.67 & $22.0(5.7)$ & 0.14 & $19.9(2.2)$ & $\mathrm{L}$ \\
\hline \multirow[t]{2}{*}{ S, Swing (\% GC) } & PR & $40.4(3.0)$ & 0.25 & $41.8(4.3)$ & 0.90 & $41.9(4.4)$ & 0.87 & $41.7(2.3)$ & $\mathrm{R}$ \\
\hline & SO & $39.6(3.9)$ & 0.53 & $40.4(3.9)$ & 0.34 & $41.6(3.9)$ & 0.44 & $40.8(1.9)$ & $\mathrm{L}$ \\
\hline \multicolumn{10}{|l|}{ Dynamic range of motion } \\
\hline \multirow[t]{2}{*}{ Hip ROM $\left(^{\circ}\right)$} & PR & $12.4(6.2)$ & 0.33 & $13.3(6.3)$ & 0.05 & $16.3(5.2)$ & 0.03 & $19.2(4.4)$ & $\mathrm{R}$ \\
\hline & SO & $18.6(4.2)$ & 0.14 & $20.2(4.9)$ & 0.22 & $19.0(4.6)$ & 0.36 & $19.6(5.0)$ & $\mathrm{L}$ \\
\hline \multirow[t]{2}{*}{ Knee ROM $\left(^{\circ}\right)$} & PR & $37.9(7.3)$ & 0.04 & $41.8(6.8)$ & 0.46 & $41.5(8.6)$ & 0.46 & $41.8(4.3)$ & $\mathrm{R}$ \\
\hline & SO & $43.2(6.4)$ & 0.42 & $42.8(7.3)$ & 0.45 & $43.1(6.4)$ & 0.44 & $42.8(4.3)$ & $\mathrm{L}$ \\
\hline
\end{tabular}

Abbreviations: PR: prosthetic side; SO: sound side; R: right side; L: left side; GC, gait cycle; ROM, range of motion.

Values are mean (SD) over the population.

Significant differences are highlighted in bold $(P<0.05)$.

a Comparison 3 vs. 6 months.

b Comparison 6 vs. 12 months.

c Comparison 12 months vs. controls. 

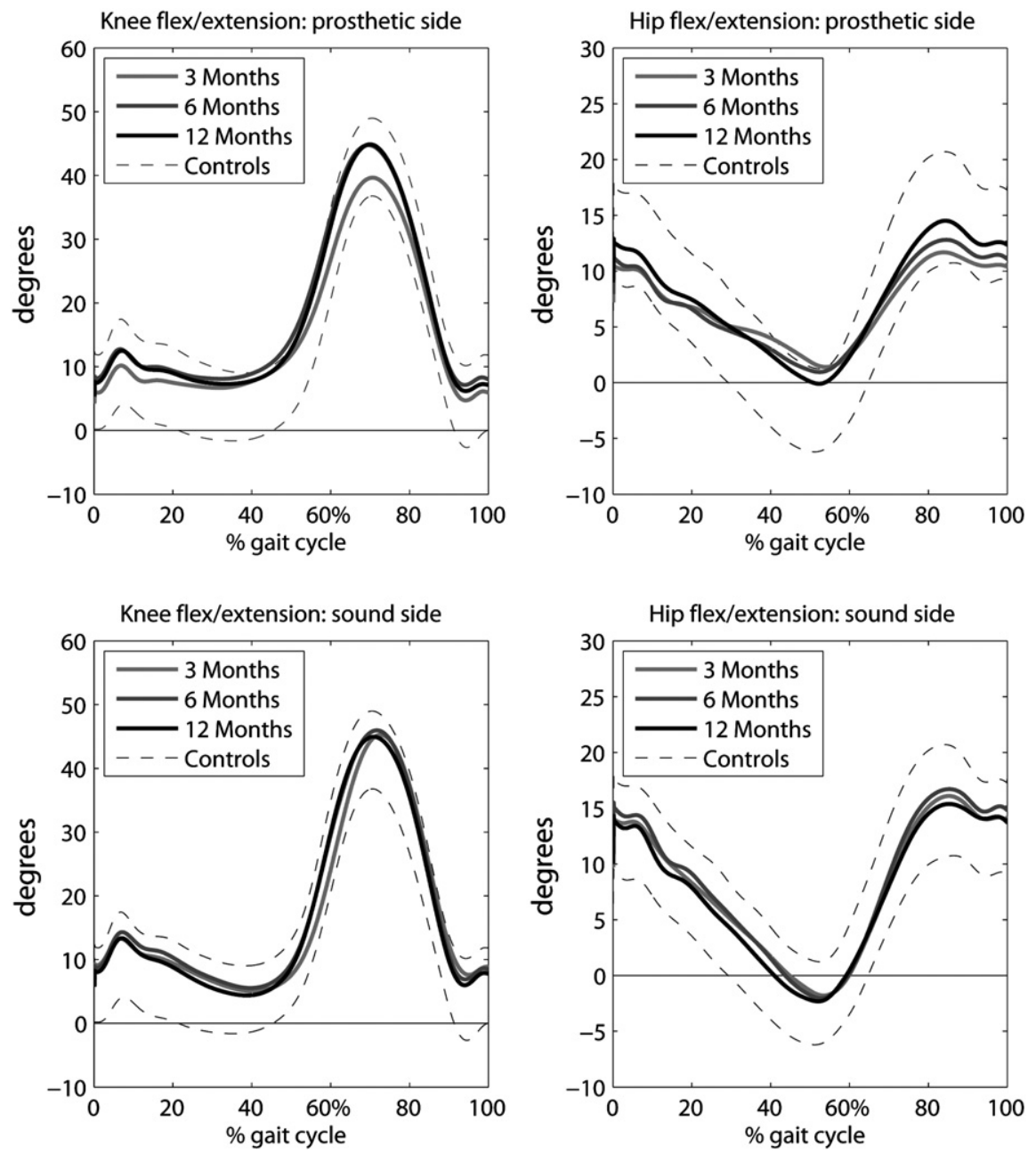

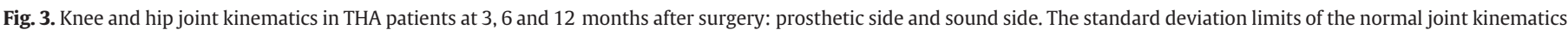
are also shown (dashed line).

anterior, rectus femoris, biceps femoris and gluteus medius the most frequent patterns showed 2 to 3 activations, in gastrocnemius lateralis the most representative patterns showed 1 to 2 activations.

\section{Tibialis Anterior}

Compared to the control group the THA group (prosthetic side) progressively anticipated the onset time of tibialis anterior during the follow up, in swing ( 2 activations) and in both pre-swing and swing (3 activations). This behavior was observed, to a minor extent, also in the sound side.

\section{Gastrocnemius Lateralis}

In the 2-activation pattern, the THA group (prosthetic side) showed a delay in the offset time of the 1st activation, compared to the control group, and a noticeable delay in the offset time of the 2nd activation, which drifted away from normality during the follow-up.

\section{Rectus Femoris}

No differences were found between groups.

\section{Biceps Femoris}

For both biceps femoris and gluteus medius, the THA group (prosthetic side) showed a prolonged activity in the main burst, compared to the control group. This indicated a higher muscular engagement of the thigh in mid-stance. In the 3-activation pattern, the second burst of activity progressively anticipated its onset during the follow-up.

\section{Gluteus Medius}

Similarly to the biceps femoris, the main burst of activity was prolonged in the prosthetic side of THA patients compared to the control group. In the 3-activation pattern, the second activation aimed at hip joint abduction - was postponed to the phase of initial swing, while in controls it occurred in terminal stance. In the sound side, a similar behavior was observed 12 months postoperatively.

\section{Discussion}

Atypical Cycles, Spatio-Temporal Parameters, Gait Phases and Joint Kinematics

THA patients showed a reduced gait velocity 3 months after surgery, but then they reached normal values. Literature reported wide evidence of velocity reduction in hip prosthetic patients [26], as well as of improvements in the follow-up [5-10]. However, in some cases it was reported that patients never reached normal values, even years after surgery [27].

In literature, the gait cycle is most often divided into two phases only, stance and swing, thus neglecting the sub-phases of stance. However, splitting stance into heel contact, flat foot contact and push off allowed us to demonstrate the prolonged duration of the heel contact in the THA group. This prolonged H-phase supports the hypothesis that the loading response was a "critical" gait phase even 1 year after surgery. Moreover, this suggests an alteration of the ankle sagittal kinematics during the weight acceptance task, as previously 


\section{Prosthetic side}

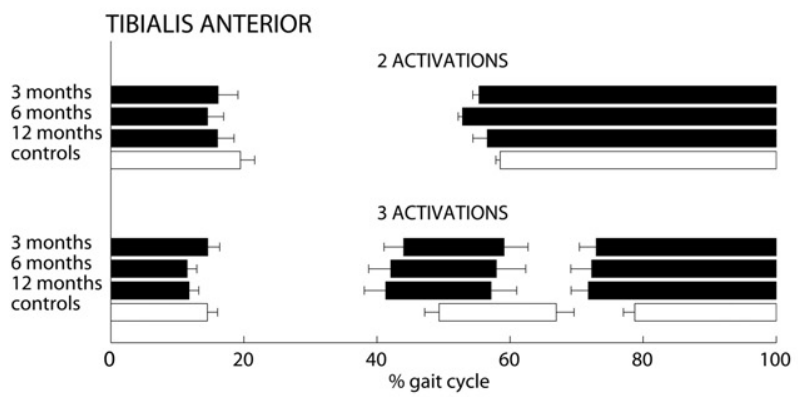

\section{GASTROCNEMIUS LATERALIS}

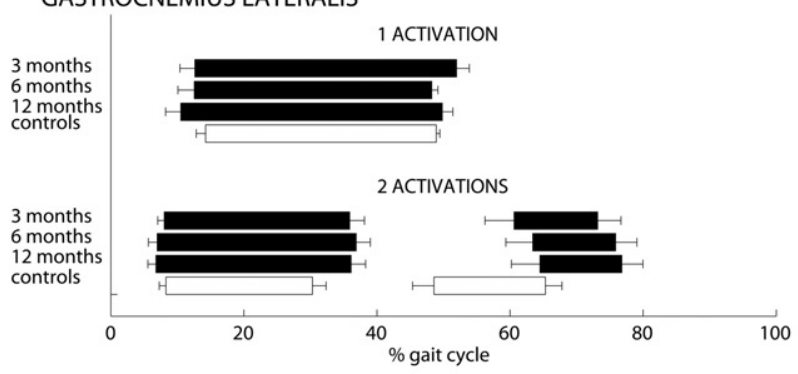

RECTUS FEMORIS
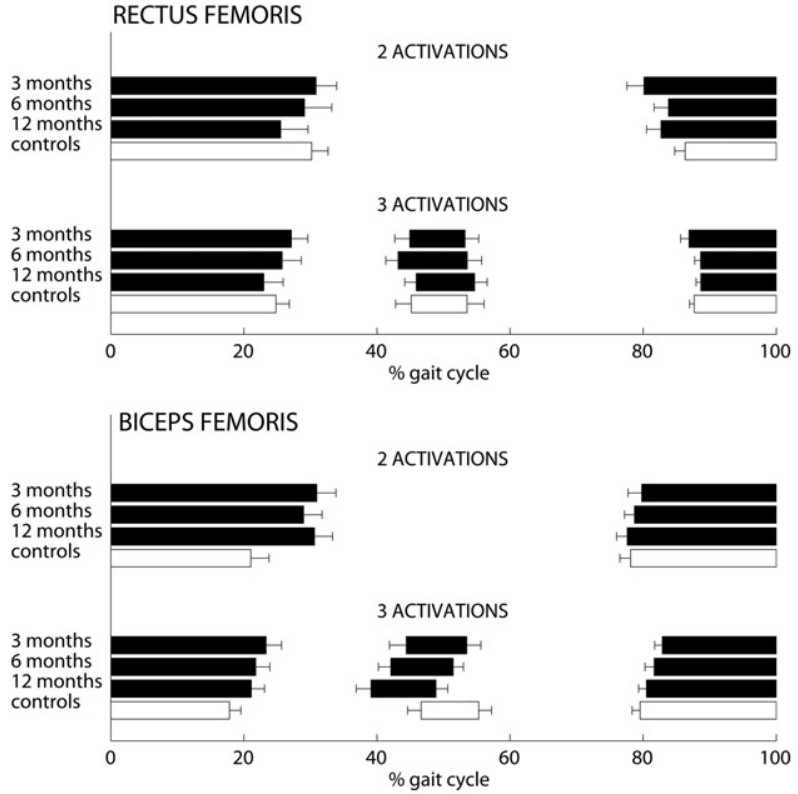

GLUTEUS MEDIUS

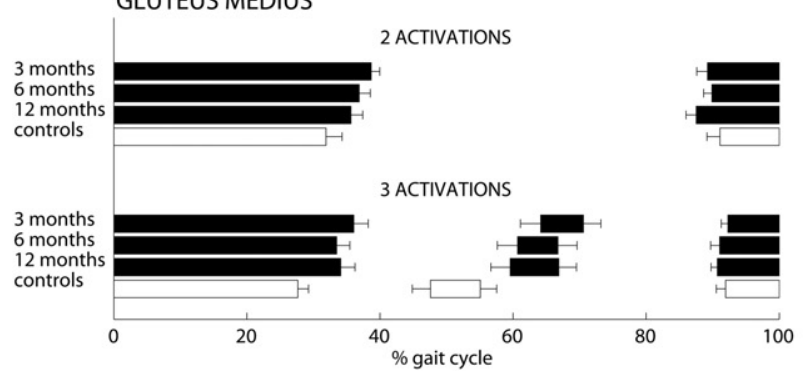

\section{Sound side}

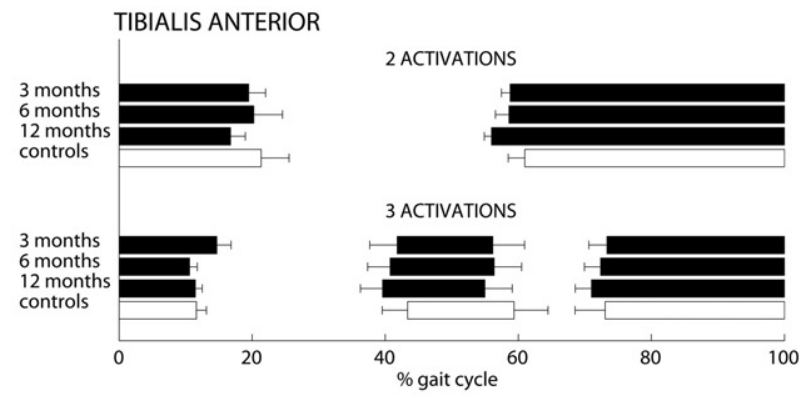

GASTROCNEMIUS LATERALIS

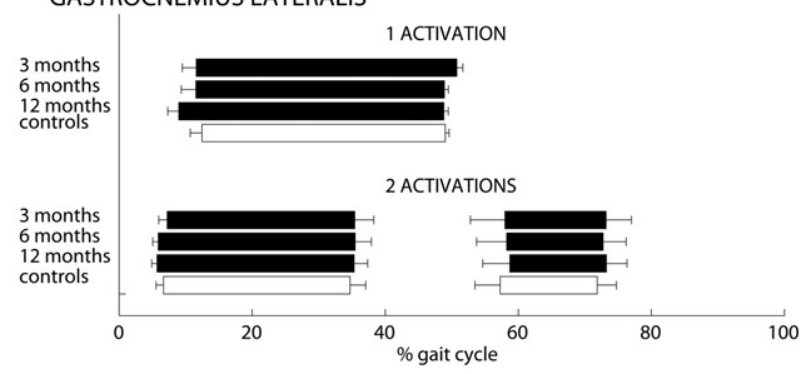

RECTUS FEMORIS
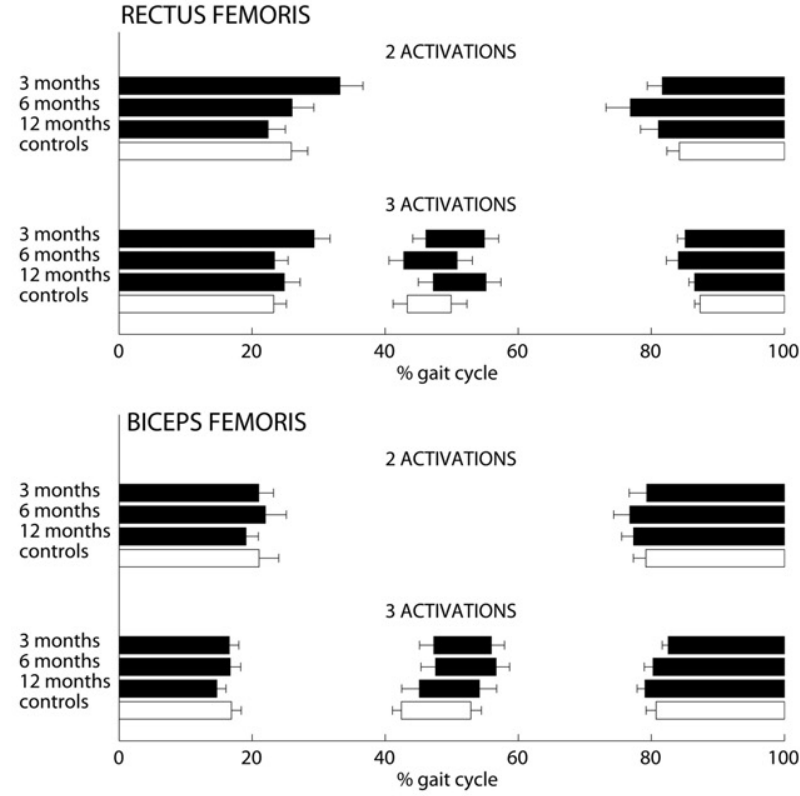

GLUTEUS MEDIUS

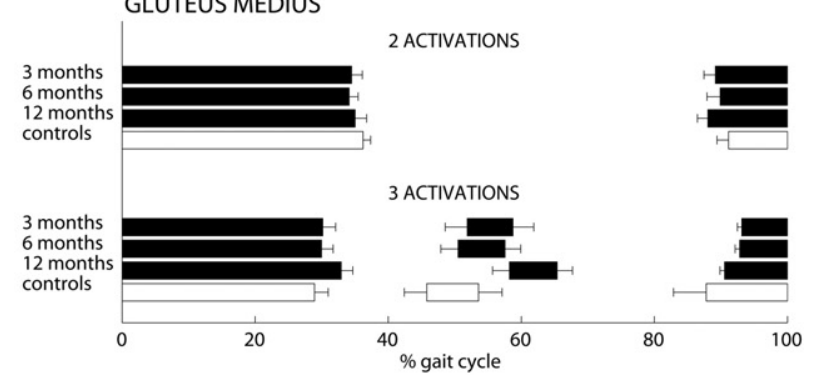

Fig. 4. EMG onset/offset activation timing (mean over the sample and standard error). The two most representative muscle activation patterns are displayed for tibialis anterior, gastrocnemius lateralis, rectus femoris, biceps femoris and gluteus medius.

reported by Beaulieu et al [28]. The diminished P-phase probably was aimed at compensating the prolonged $\mathrm{H}$-phase.

In accordance with previous studies, the sagittal-plane dynamic ROM of the affected hip improved considerably during the postoperative follow-up, but it did not reach normality $[28,29]$.
The alterations observed in the hip and knee kinematics suggest that the prosthetic limb was more flexed during stance, which could explain the early onset of the tibialis anterior activity in preparation for initial swing, where increased dorsiflexion would be needed. 
This study demonstrated that THA patients showed more atypical cycles than controls. This aspect did not improve during the followup. The presence of a greater number of atypical cycles may be explained by many concurrent factors, among which are strength deficits and dynamic joint stiffness [4,29]. We hypothesized that another important factor may be a diminished proprioception after THA. In fact, after hip surgery there are a complete loss of the joint capsule and capsule ligaments, and a partial loss of extra-capsular mechanoreceptors, such as stretch receptors in the adjacent tendons and muscles. Both capsular and extra-capsular components are known to be involved in proprioception and in joint-position sense, even if there is a debate in literature on the relative importance of these components $[2,30]$.

Although the THA patients increased their velocity and knee dynamic ROM between 3 and 6 months after surgery, no other significant improvements were observed between 6 and 12 months, and the percentage of atypical cycles, H-phase, P-phase and hip dynamic ROM did not reach normal values 12 months after surgery. Our results suggest that rehabilitation protocols should not only focus on the first few months after surgery, but continue in a long-term effort to normalize gait by muscle strengthening and motor relearning, as observed by Hodt-Billington et al [5].

\section{Muscle Activation}

In literature, there are only a few THA gait studies focusing on EMG data. Vogt et al [14] studied the muscle activation pattern of gluteus medius, but the evaluation was performed during treadmill ambulation, which is not directly comparable with overground walking [31]. A study on the thrust plate prosthesis reported an increased activity of the gluteus maximus, medius and the tensor fascie latae on the operated side, while no changes were reported in the activity of the adductor longus, rectus femoris and biceps femoris, bilaterally, with respect to controls [12]. The prolonged timing of the muscle activity bursts that we found for gluteus medius is in accordance with previous findings [12]. Furthermore, we observed another burst of activity for gluteus medius: in controls this burst appeared between terminal stance and pre-swing (around $50 \% \mathrm{GC}$ ), but in THA patients it was delayed up to the swing phase (around 65\% GC), possibly indicating a strategy of hip abduction when the hip was unloaded. The gluteus medius of the sound side also showed this abnormal activation pattern, but only later in the follow-up (12 months postoperatively), possibly indicating an arising compensative strategy aimed at improving gait symmetry. We discussed above that the increased hip and knee flexion, along with the earlier activation of the tibialis anterior (prior to initial swing), suggested the need to prepare for foot clearance in swing. This strategy may have been continued in early swing via circumduction of the swing limb (with gluteus medius activity), to avoid ground contact.

\section{Limitations of the Present Work}

A number of important limitations regarding the present study need to be acknowledged. First, we didn't collect preoperative data and hence the effect of THA is unknown. Preoperative data would have been important to evaluate the presence of an antalgic gait, and to evidence e.g. Trendelenburg gait or short leg syndrome.

A second limitation of the study is the lack of a control group with a comparable pathology, i.e. hip osteoarthritis not undergoing surgery. The presence of such a control group would have allowed us to analyze the gait differences between the THA patients and a nonsurgical treatment group.

Full load was gained by patients $15-30$ days before the 3-month gait test. We consider this period of time sufficient for a (first) patient's reconditioning and a (partial) recovery of gait. However, it could be possible that protected weight bearing carried out during the rehabilitation period could bias the 3-month gait test and hence the 3vs. 6-month comparison. Furthermore, it should be noted that the presence of post-operative pain might result in an antalgic gait that could also bias the analysis. However, none of our patients reported persistent pain post-operatively in such a way as to limit their gait. Three patients reported mild pain having no effect on average activities, and rarely moderate pain with unusual activities, at three months post-operatively.

Another limitation of the work is the fact that some of the variables that have been considered postoperatively might be influenced by the reconditioning that occurs secondary to the underlying pathology. In this respect, caution should be taken in the interpretation of the results.

\section{Conclusions}

The results of the present study revealed a residual impairment of the prosthetic side and compensation mechanisms arising in the sound side at one year post-operatively. The study also revealed an augmented number of atypical cycles. The EMG analysis demonstrated the presence of abnormal patterns of muscle activation that did not normalize during follow-up. These anomalies did not affect only the muscles damaged by surgery, such as the gluteus medius, but also the other leg muscles, evidencing changes in the overall walking scheme.

More research should be devoted to understand if a targeted rehabilitation could be beneficial in improving patient function and allowing a more symmetrical gait.

\section{Acknowledgments}

The authors wish to thank Rosanna Evangelisti, P.T., and Laura Rivella, M.D., in charge coordinator and former head of Struttura Complessa Recupero e Rieducazione Funzionale, ASLTO4 Piemonte, Ivrea (TO), Italy.

\section{References}

1. Siopack J, Jergesen H. Total hip arthroplasty. West J Med 1995;162:243.

2. Grigg P, Finerman GA, Riley LH. Joint-position sense after total hip replacement. J Bone Joint Surg 1973;55:1016.

3. Baker AS, Bitounis VC. Abductor function after total hip replacement. An electromyographic and clinical review. J Bone Joint Surg [Br] 1989;71:47.

4. Downing ND, Clark DI, Hutchinson JW, et al. Hip abductor strength following total hip arthroplasty: a prospective comparison of the posterior and lateral approach in 100 patients. Acta Orthop Scand 2001;72:215

5. Hodt-Billington C, Helbostad JL, Vervaat W, et al. Changes in gait symmetry, gait velocity and self-reported function following total hip replacement. J Rehabil Med 2011;43:787.

6. Bennett D, Ogonda L, Elliott D, et al. Comparison of gait kinematics in patients receiving minimally invasive and traditional hip replacement surgery: a prospective blinded study. Gait Posture 2006;23:374.

7. Madsen MS, Ritter MA, Morris HH, et al. The effect of total hip arthroplasty surgical approach on gait. J Orthop Res 2004;22:44.

8. Mayr E, Nogler M, Benedetti MG et al. A prospective randomized assessment of earlier functional recovery in THA patients treated by minimally invasive direct anterior approach: a gait analysis study. Clin Biomech (Bristol Avon) 2009;24:812.

9. Mont MA, Seyler TM, Ragland PS, et al. Gait analysis of patients with resurfacing hip arthroplasty compared with hip osteoarthritis and standard total hip arthroplasty.J Arthroplasty 2007;22:100.

10. Nantel J, Termoz N, Vendittoli PA, et al. Gait patterns after total hip arthroplasty and surface replacement arthroplasty. Arch Phys Med Rehabil 2009:90:463.

11. Shrader MW, Bhowmik-Stoker M, Jacofsky MC, et al. Gait and stair function in total and resurfacing hip arthroplasty. A pilot study. Clin Orthop Relat Res 2009; 467:1476.

12. Steens W, Rosenbaum D, Goetze C, et al. Clinical and functional outcome of the Thrust Plate Prosthesis: short- and medium-term results. Clin Biomech (Bristol Avon) 2003;18:647.

13. Perron $\mathrm{M}$, Malouin $\mathrm{F}$, Moffet $\mathrm{H}$, et al. Three-dimensional gait analysis in women with a total hip arthroplasty. Clin Biomech (Bristol Avon) 2000;15:504

14. Vogt LBW, Pfeifer K, Galm R. Muscle activation pattern of hip arthroplasty patients in walking. Res Sports Med 2005;12:191.

15. Gotze C, Rosenbaum D, Hoedemaker J, et al. Is there a need of custom-made prostheses for total hip arthroplasty? Gait analysis, clinical and radiographic analysis of customized femoral components. Arch Orthop Trauma Surg 2009; 129:267. 
16. Agostini V, Nascimbeni A, Gaffuri A, et al. Normative EMG activation patterns of school-age children during gait. Gait Posture 2010;32:285.

17. Agostini V, Knaflitz M. Statistical gait analysis. In: Acharya RU, Molinari F, Tamura T, Naidu DS, Suri JS, editors. Distributed diagnosis and home healthcare $\left(\mathrm{D}_{2} \mathrm{H}_{2}\right), \mathrm{Vol} .2$. Stevenson Ranch: American Scientific Publishers; 2012. p. 99.

18. Agostini V, De Luca R, Corgiat Mansin L, et al. Reduction of gait abnormalities in type 2 diabetic patients due to physical activity: a quantitative evaluation based on statistical gait analysis. J Mech Med Biol 2012;12(5):1.

19. Foucher KC, Hurwitz DE, Wimmer MA. Preoperative gait adaptations persist one year after surgery in clinically well-functioning total hip replacement patients. J Biomechanics 2007; 40:3432.

20. Harris WH. Traumatic arthritis of the hip after dislocation and acetabular fractures: treatment by mold arthroplasty. J Bone Joint Surg Am 1969;51:737.

21. Beattie P, Isaacson K, Riddle DL, et al. Validity of derived measurements of leglength differences obtained by use of a tape measure. Phys Ther 1990;70:150

22. Winter DA. The biomechanics and motor control of human gait: normal, elderly and pathological. 2nd ed. Waterloo, Ont: University of Waterloo Press; 1991. p. 57.

23. Bonato P, D'Alessio T, Knaflitz M. A statistical method for the measurement of muscle activation intervals from surface myoelectric signal during gait. IEEE Trans Biomed Eng 1998;45:287.
24. Di Nardo F, Fioretti S. Statistical analysis of surface electromyographic signal for the assessment of rectus femoris modalities of activation during gait. J Electromyo Kines 2013;23(1):56.

25. Krzanowski WJ. Principles of multivariate analysis: a user's perspective. Oxford (UK): Clarendon Press; 1988

26. Ewen AM, Stewart S, St Clair Gibson A, et al. Post-operative gait analysis in total hip replacement patients-a review of current literature and meta-analysis. Gait Posture 2012;36:1.

27. Kyriazis V, Rigas C. Temporal gait analysis of hip osteoarthritic patients operated with cementless hip replacement. Clin Biomech (Bristol Avon) 2002;17:318.

28. Beaulieu ML, Lamontagne M, Beaulé PE. Lower limb biomechanics during gait do not return to normal following total hip arthroplasty. Gait Posture 2010; $32: 269$.

29. Tateuchi H, Tsukagoshi R, Fukumoto Y, et al. Dynamic hip joint stiffness in individuals with total hip arthroplasty: relationships between hip impairments and dynamics of the other joints. Clin Biomech (Bristol Avon) 2011;26(6):598

30. Ishii $Y$, Tojo $T$, Terajima $K$, et al. Intracapsular components do not change hip proprioception. J Bone Joint Surg [Br] 1999;81B:345.

31. Lee SJ, Hidler J. Biomechanics of overground vs. treadmill walking in healthy individuals. J Appl Physiol 2008;104:747. 